\title{
Prepregnancy Weight, Gestational Weight Gain, and Risk of Growth Affected Neonates
}

\author{
Tiffany A. Moore Simas, M.D., M.P.H., M.Ed., ${ }^{1,2,3}$ Molly E. Waring, Ph.D., \\ Xun Liao, M.S., Anne Garrison, M.D., ${ }^{1,3}$ Gina M.T. Sullivan, M.D., \\ Allison E. Howard, B.S., ${ }^{3}$ and Janet R. Hardy, Ph.D., M.Sc., M.P.H. ${ }^{1,2,5}$
}

\begin{abstract}
Background: In 2009, the Institute of Medicine published revised gestational weight gain (GWG) guidelines with changes notable for altered body mass index (BMI) categorization as per World Health Organization criteria and a stated range of recommended gain (11-20 pounds) for obese women. The goal of this study was to evaluate associations between maternal BMI-specific GWG adherence in the context of these new guidelines and risk of small for gestational age (SGA) and large for gestational age (LGA) neonates.

Methods: Subjects were a retrospective cohort of 11,203 live birth singletons delivered at 22-44 weeks at a Massachusetts tertiary care center between April 2006 and March 2010. Primary exposure was GWG adherence (inadequate, appropriate, or excessive) based on BMI-specific recommendations. SGA and LGA were defined as $<10$ th and $\geq 90$ th percentiles of U.S. population growth curves, respectively. The association between GWG adherence and SGA and LGA was examined in polytomous logistic regression models that estimated adjusted odds ratios (AOR) stratified by prepregnancy weight status, controlling for potential confounders.

Results: Before pregnancy, 3.8\% of women were underweight, $50.9 \%$ were normal weight, $24.6 \%$ were overweight, and $20.6 \%$ were obese. Seventeen percent had inadequate GWG, and 57.2\% had excessive GWG. Neonates were 9.6\% SGA and 8.7\% LGA. Inadequate GWG was associated with increased odds of SGA (AOR 2.51, 95\% confidence interval [CI] 1.31-4.78 for underweight and AOR 1.78, 95\% CI 1.42-2.24 for normal weight women) and decreased odds of LGA (AOR 0.5, 95\% CI 0.47-0.73 for normal weight and AOR 0.56, 95\% CI 0.34-0.90 for obese women). Excessive GWG was associated with decreased odds of SGA (AOR 0.59, 95\% CI 0.47-0.73 for normal weight and AOR 0.64, 95\% CI 0.47-0.89 for overweight women) and increased odds of LGA (AOR 1.76, 95\% CI 1.38-2.24 for normal weight, AOR 2.99, 95\% CI 1.92-4.65 for overweight, and AOR 1.55, 95\% CI 1.10-2.19 for obese women).

Conclusions: Efforts to optimize GWG are essential to reducing the proportion of SGA and LGA neonates, regardless of prepregnancy BMI.
\end{abstract}

\section{Introduction}

B OTH SMALL FOR GESTATIONAL AGE (SGA) AND LARGE FOR GESTATIONAL AGE (LGA) neonates are at considerable immediate and long-term health risk. SGA neonates are at risk for low Apgar scores, meconium aspiration, seizures, respiratory complications, extended hospital stays, and long-term sequelae, including metabolic syndrome and neurologic deficits. ${ }^{1,2}$ LGA neonates are at risk for cardiac anomalies, neural tube defects, shoulder dystocia, cesarean delivery, intrauterine fetal demise, neonatal intensive care unit (NICU) admissions, and long-term obesity. ${ }^{2-5}$
Both prepregnancy body mass index (BMI) and gestational weight gain (GWG) outside of recommended ranges are associated with SGA and LGA neonates. Overweight and obese women are at increased and decreased risks for LGA and SGA neonates, respectively. ${ }^{6}$ Regardless of prepregnancy weight status, women who gain excessively during pregnancy are more likely to have macrosomic infants. ${ }^{1-4}$ For normal weight women, GWG below and above 1990 Institute of Medicine (IOM) recommendations is associated with increased risk of SGA and LGA neonates, respectively. ${ }^{7}$ However, considering that $50 \%$ of pregnancies are unintended, ${ }^{8}$ for the vast majority of women, GWG is the only weight parameter that is

Departments of ${ }^{1}$ Obstetrics and Gynecology, and ${ }^{2}$ Pediatrics, Unviersity of Massachusetts Medical School, Worcester, Massachusetts. ${ }^{3}$ Department of Obstetrics and Gynecology, UMass Memorial Health Care, Worcester, Massachusetts.

Departments of ${ }^{4}$ Quantitative Health Sciences, and ${ }^{5}$ Medicine, University of Massachusetts Medical School, Worcester, Massachusetts. 
modifiable once prenatal care and associated counseling are initiated.

In May 2009, the IOM published new recommendations with regard to weight gain in pregnancy. ${ }^{9}$ The revised guidelines are notable for two changes: (1) underweight, normal weight, overweight, and obese BMI categories were changed from the Metropolitan Life Insurance Table to World Health Organization (WHO) cutoff points, ${ }^{9}$ and (2) obese women were provided with a range of recommended gain (11-20 pounds) $)^{9}$ compared to the prior recommendation of "at least 15 pounds" with an unbounded upper limit. ${ }^{10}$ Previous studies investigating associations of GWG with risk of SGA and LGA neonates have done so in the context of 1990 IOM GWG guidelines; only the recent publication by Margerison Zilko et al. ${ }^{11}$ puts this important issue in the context of the updated guidelines. The goal of this study was to estimate the association between adherence to the revised 2009 IOM prepregnancy BMI-specific GWG recommendations and the occurrence of SGA or LGA neonates.

\section{Materials and Methods}

The University of Massachusetts Medical School's clinical partner, UMass Memorial Health Care's (UMMHC) automated electronic Labor \& Delivery (L\&D EMR) export database provided data for this retrospective cohort study. The initial cohort consisted of $>16,000$ women who delivered singleton, live birth, nonanomalous neonates between April 1, 2006, and March 31, 2010 (Fig. 1). Neonates with congenital anomalies as designated within the complications section of the delivery record were excluded. Women without recorded maternal prepregnancy weight, height, or documented GWG were excluded. Women with missing or unknown demographics of interest (marital status, smoking status, maternal age, parity, and race) were excluded. Women delivering at unknown gestational age, at $<22$ weeks or $>44$ weeks, or who delivered a neonate with unknown gender or with unknown birth weight were also excluded. Finally, for women with more than one pregnancy during the study period, one pregnancy was randomly selected for inclusion in analyses. Our final sample included 11,203 women and their neonates (Fig. 1). The export database is surveyed periodically to evaluate internal consistency by direct comparison with patient charts. Variables missing information or data outliers were verified or corrected by chart review where possible. The UMMS Institutional Review Board approved this study.

Prepregnancy weight status was assessed with BMI (kilogram $/$ meter $^{2}$ ) calculated from prepregnancy weight and height. Height in the L\&D EMR was either obtained from the prenatal record or self-reported at time of delivery. Prepregnancy weight was recorded in the L\&D EMR. L\&D nurses were instructed to record prepregnancy weight according to a prioritized sequence as available: (1) self-reported prepregnancy weight as recorded in the woman's prenatal record, (2) weight self-reported by the women upon admission for delivery, or (3) measured weight at first prenatal visit as recorded in her prenatal record. Women's prepregnancy weight status was categorized according to 2009 IOM BMI categories and thus is consistent with WHO categories as follows: underweight $\left(\mathrm{BMI}<18.5 \mathrm{~kg} / \mathrm{m}^{2}\right)$, normal weight $\left(18.5 \mathrm{~kg} / \mathrm{m}^{2} \leq \mathrm{BMI}<25 \mathrm{~kg} / \mathrm{m}^{2}\right), \quad$ overweight $\quad\left(25 \mathrm{~kg} / \mathrm{m}^{2} \leq\right.$ $\left.\mathrm{BMI}<30 \mathrm{~kg} / \mathrm{m}^{2}\right)$ and obese $\left(30 \mathrm{~kg} / \mathrm{m}^{2} \leq \mathrm{BMI}\right){ }^{9}$
Similar to prepregnancy weight, GWG was ascertained in L\&D EMR through one of two sources per availability: (1) selfreport at time of delivery admission or (2) documented weight at last prenatal visit subtracted from prepregnancy weight in prenatal records. GWG was categorized as inadequate, appropriate, or excessive based on the IOM's 2009 prepregnancy BMI-specific GWG guidelines. Appropriateness of women's GWG was categorized using recommended GWG corresponding to individual gestational age at delivery, thus allowing for inclusion of preterm deliveries in addition to fullterm deliveries. To account for gestational age at delivery, minimum and maximum recommended gains at each week of gestational age were determined using IOM recommendations of gain by the end of the first trimester (week 13) and trajectory of gain throughout the second and third trimesters, assuming the following ranges of gain achieved by the 40th week: 28-40 pounds for women who were underweight before pregnancy, 25-35 pounds for women of normal weight, $15-25$ pounds for overweight women, and 11-20 pounds for obese women. ${ }^{9}$

Neonates were considered to beSGA and LGA, respectively, if birth weight was $<10 \mathrm{th}^{12}$ and $\geq 90 \mathrm{th}^{13}$ percentile of 19992000 U.S. national reference data ${ }^{14}$ for singletons, accounting for gestational age and gender. ${ }^{11}$ Gestational age at delivery was based on best dates for estimated date of confinement as per clinician evaluation and as recorded in L\&D EMR.

Other characteristics abstracted from the L\&D EMR and considered as covariates were (1) maternal age ( $\leq 19$ years, 20-24 years, $25-39$ years, 30-34 years, $\geq 35$ years), (2) marital status (married vs. unmarried, including single, divorced, widowed, and separated), (3) ever smoker, which included smoking before or any time during pregnancy, (4) parity (nulliparous, primiparous, multiparous), (5) race/ethnicity representing either self-reported or attributed race/ethnicity (white, Hispanic, black, Asian, other race/ethnicity), (6) hypertension diagnosis (prepregnancy or pregnancy-associated hypertension diagnosis vs. absence of any hypertensive disease diagnosis), and (7) diabetes status (pregestational or gestational diabetes mellitus [GDM] diagnosis vs. absence of any diabetes diagnosis).

\section{Statistical analyses}

Demographic summary statistics are presented as either mean \pm standard deviation (SD) for continuous variables or as frequency measures for categorical variables. We initially included women of any prepregnancy weight status in our analyses and tested one-way interactions between covariates using chi-square tests. The interaction between prepregnancy weight status and adherence to GWG recommendations was statistically significant. Therefore, we stratified our analyses by prepregnancy weight status in order to make our results more easily interpreted in the context of providing useful and effective prenatal counseling.

We estimated odds ratios (ORs) and 95\% confidence intervals (CIs) for SGA and LGA in relation to adherence to GWG recommendations (GWG within recommended ranges compared to inadequate or excessive GWG), stratified by prepregnancy weight status, using polytomous logistic regression models. In polytomous logistic regression, the outcome takes one of three or more categories, and models are simultaneously fit using maximum likelihood to estimate ORs for each group compared to a common reference group. ${ }^{15} \mathrm{We}$ 
FIG. 1. Study flow diagram. BMI, body mass index; GA, gestational age; GWG, gestational weight gain; L\&D EMR, labor and delivery electronic medical record; LGA, large for gestational age; SGA, small for gestational age.

\section{University of Massachusetts Medical School \\ UMass Memorial Health Care \\ L\&D EMR \\ Export Relational Database}

April 1, 2006 - March 31, 2010

Total Births $=17,217$

(Total Deliveries $=16,700$ )

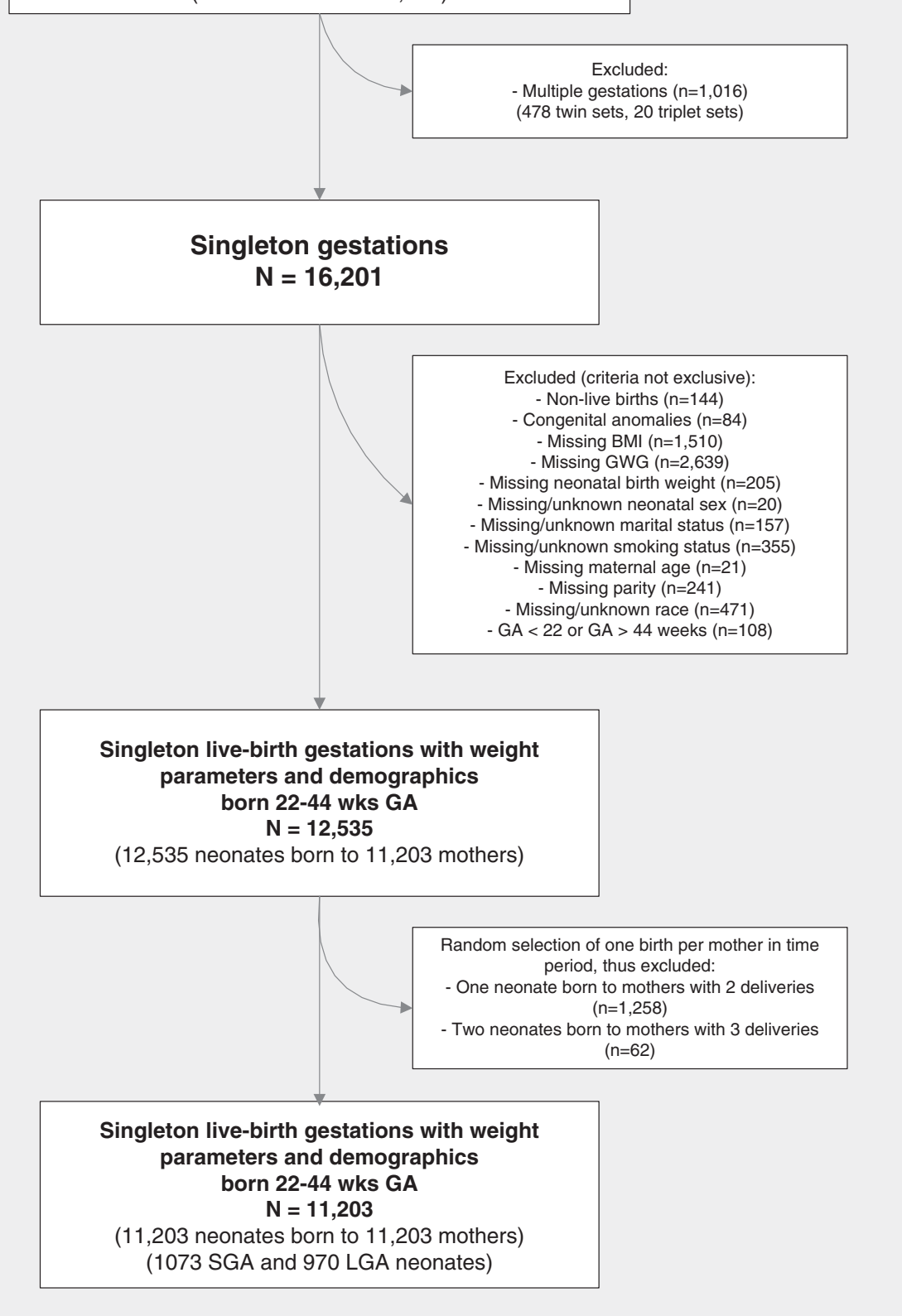

controlled for confounding using multivariate adjustment. Covariates were considered for inclusion in the regression models based on previous research examining GWG and SGA/LGA, 1,2,4,6,7,11,16,17 availability in the dataset, and significance on bivariate comparisons. We initially included in multivariate-adjusted models those covariates that were significantly related to appropriateness of neonatal size for gestational age category (SGA, appropriate for gestational age
[AGA], LGA) at $p<0.10$ within strata of prepregnancy BMI. Adjusted models revealed significant correlation of age with parity or marital status on SGA, AGA, and LGA categories; thus, age was not included in the final adjusted models.

We also performed sensitivity analyses through examination of several other models. For women $<20$ years of age at delivery, we calculated age-specific and gender-specific $\mathrm{BMI}$ percentiles and defined BMI categories according to the 
Centers for Disease Control and Prevention (CDC) definitions for children: underweight $(<5$ th percentile), healthy weight ( $\geq 5$ th and $<85$ th percentile), overweight $(\geq 85$ th and $<95$ th percentile), and obese ( $\geq 95$ th percentile). ${ }^{18}$ For these analyses, at risk of overweight was considered overweight, and overweight was considered obese with regard to consideration of recommended GWG ranges. We additionally examined models in which adolescents were excluded. As the definitions of SGA and LGA are percentiles based on population growth curves, and as infant birth weights have changed over time, models using different U.S. national reference data, ${ }^{19}$ controlling for gender, were considered. Models in which mothers with diabetes or hypertension were excluded from analyses were evaluated because the relationship between these conditions and GWG is complex and may be on the causal pathway; for example, women with GDM may have lower GWG as a result of interventions for their diabetes. Additionally, this dataset does not adequately allow for distinction between pregestational and gestational diagnoses regarding these disease processes. Finally, preterm deliveries were excluded in one set of models, as reference cutoff points for SGA and LGA are less reliably measured by fetal growth reference population curves because preterm births are biased by a significant portion of preterm neonates being growth restricted. Analyses were performed using SAS (version 9.2, SAS Institute Inc., Cary, NC) statistical analysis software.

\section{Results}

Before pregnancy, $3.8 \%$ of women were underweight, $50.9 \%$ were normal weight, $24.6 \%$ were overweight, and $20.6 \%$ were obese. Nearly $75 \%$ of women gained outside of the ranges recommended by the IOM $(17.0 \%$ gained inadequately and $57.2 \%$ gained excessively). Women were predominantly white $(63.5 \%)$, married $(60.1 \%)$, multiparous $(58.6 \%)$, and nonsmokers $(87.5 \%)$. Women were, on average, aged $29.0( \pm 6.1)$ years. The most common comorbidities were pregestational diabetes or GDM $(6.2 \%)$ and pregestational or pregnancy-related hypertensive disease $(8 \%)$. Women were delivered at a mean gestational age of $38.9( \pm 2.3)$ weeks, and their neonates had mean birth weights of $3310.5( \pm 620.0) \mathrm{g}$. Approximately one tenth of neonates were SGA $(9.6 \%$, $n=1073)$, and $8.7 \%$ were LGA $(n=970)$.

Table 1 displays demographics specific to combined BMI and GWG adherence categories. Within each BMI category, when progressing from inadequate to appropriate to excessive gain, infant birth weight increased (Table 1). In each BMI category, frequency of hypertensive disease diagnoses was highest among women who gained excessively, and frequency of preterm delivery was highest among women who gained inadequately (Table 1).

Within BMI categories, when progressing from inadequate to appropriate to excessive gain, frequency of SGA decreased and frequency of LGA increased, respectively (Fig. 2). Regardless of prepregnancy weight status, frequency of SGA was lower with higher GWG; additionally, frequency of LGA was higher among women of higher prepregnancy BMI who experienced greater weight gain. Underweight women with inadequate GWG had the highest frequency of SGA neonates. Obese women who gained excessively had the highest frequency of LGA neonates (Fig. 2).
Inadequate GWG was associated with increased adjusted odds of SGA among underweight and normal weight women (Table 2). Excessive GWG was associated with decreased adjusted odds of SGA among normal weight and overweight women, with trends for decreased odds in underweight and obese women. Inadequate GWG was associated with decreased adjusted odds of LGA among normal weight and obese women, with trends for decreased odds in overweight women. Excessive GWG was associated with increased adjusted odds of LGA among normal weight, overweight, and obese women, with trends for increased odds in underweight women. It should be noted that confidence intervals are likely wide in the underweight group because of relatively small numbers of women in this BMI category. The results of the various sensitivity analyses were very similar to those of the main analyses (data not shown).

\section{Discussion}

Inadequate GWG was associated with increased odds of SGA neonates, and excessive gain was associated with increased odds of LGA neonates. Conversely, inadequate gain was associated with decreased odds of LGA neonates, and excessive gain was associated with decreased odds SGA neonates. Using 1990 IOM guidelines, previous published studies have reported associations between maternal prepregnancy weight, ${ }^{20}$ maternal BMI, ${ }^{1,21}$ and $\mathrm{GWG}^{1,17,20}$ with fetal growth. A recent review of 35 studies found strong evidence supporting an association between excessive GWG and LGA and between inadequate GWG and SGA. ${ }^{22}$ Our findings contribute to the limited information available on this topic specific to the new 2009 IOM GWG guidelines and thus describe the contemporary burden of growth-affected neonates born to women gaining outside of currently recommended GWG ranges. Margerison Zilko et al. ${ }^{11}$ is the only other group to date to have published study findings based on the 2009 revised IOM guidelines for GWG and neonatal outcomes of SGA or LGA. In the current study, we found similar significant associations between adherence to GWG recommendations and SGA and LGA neonates, independent of maternal demographic and pregnancy characteristics. Margerison Zilko et al. ${ }^{11}$ evaluated the odds of SGA and LGA by prepregnancy BMI and GWG adherence in separate adjusted models. Noting an interaction between BMI and GWG adherence, however, we presented our results stratified by maternal prepregnancy weight status, thus highlighting the increased risk of SGA and LGA in a manner directly relevant to provider counseling about GWG.

Two studies ${ }^{20,21}$ have reported increasing trends in LGA, and a third study reported decreased birth weight (mean $52 \mathrm{~g}$ ) among all singleton term neonates in the U.S. between 1990 and $2005 .{ }^{16}$ This last study additionally reported that LGA rates had decreased to $8.9 \%$, consistent with our findings $(8.7 \%)$, and reported stable SGA rates of approximately $10.2 \% .{ }^{16}$ In our study, approximately one tenth $(9.6 \%)$ of neonates were SGA.

It should be noted that our cohort spans the change between the 1990 and 2009 guidelines. As the American College of Obstetricians and Gynecologists (ACOG) had not yet clearly endorsed the 2009 guidelines, counseling practices likely did not change in the transition period, and most women had already delivered or were pregnant when the IOM published its updated guidelines in 2009. Thus, the term 


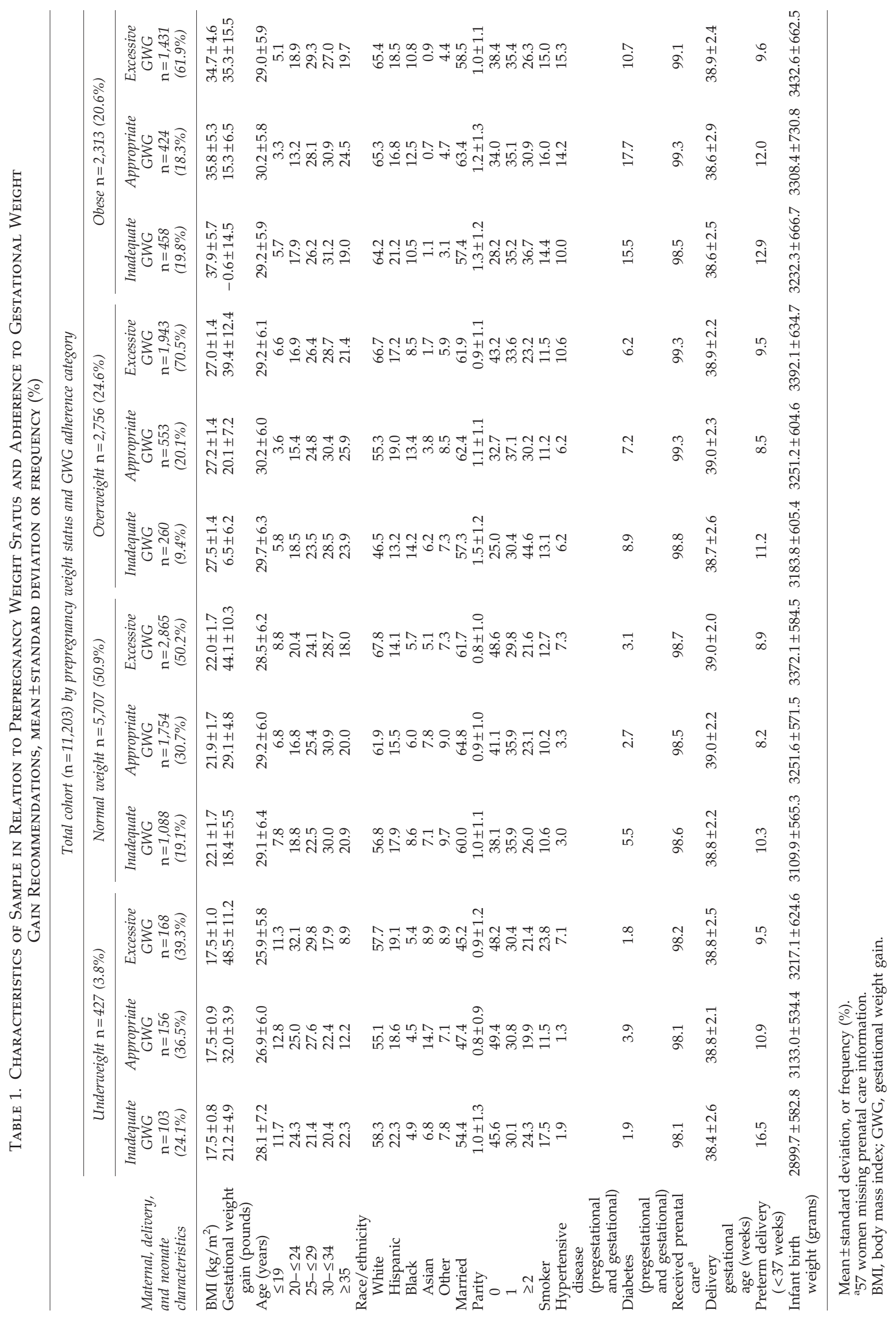



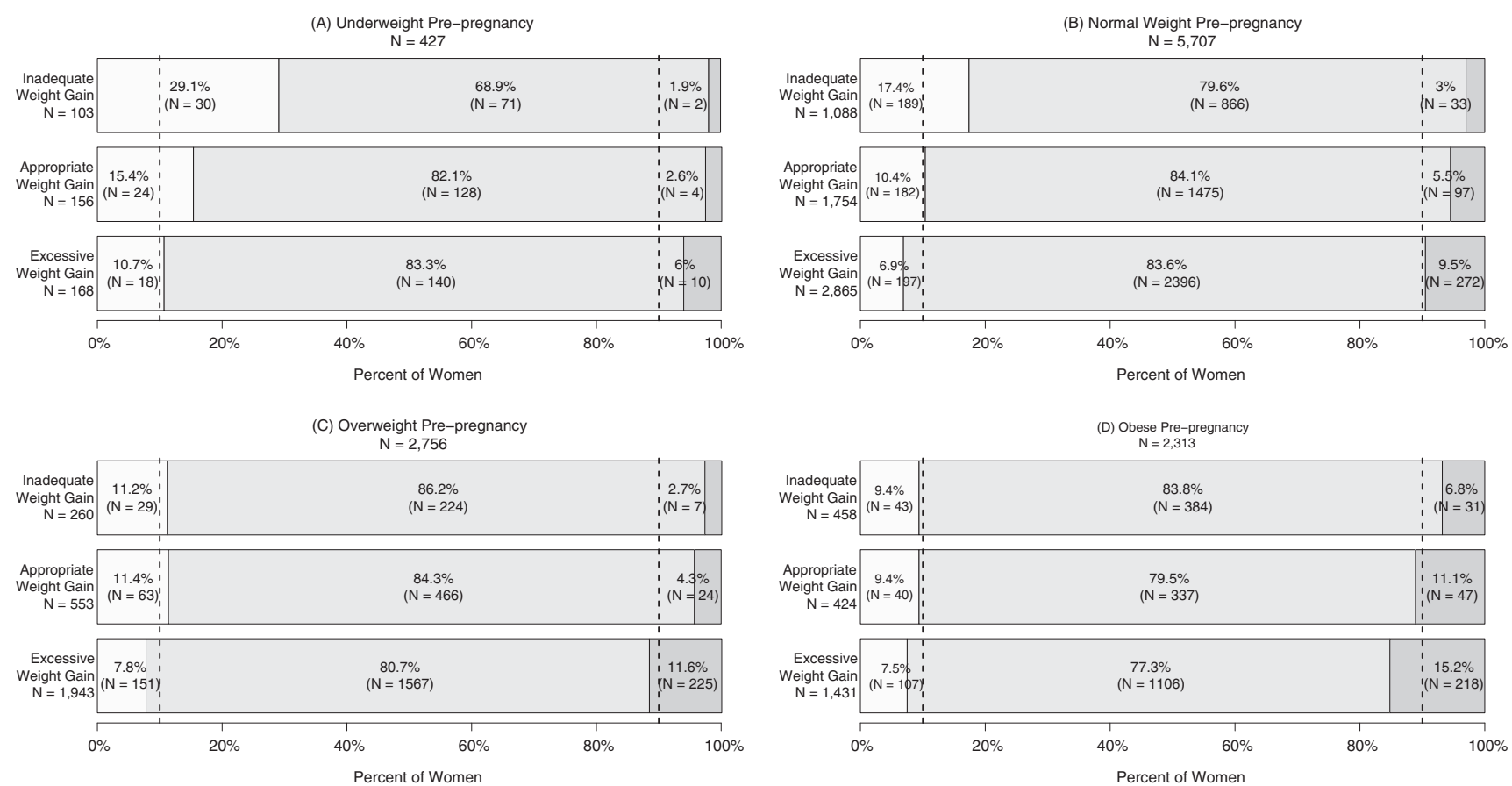

FIG. 2. Distribution of small, appropriate, and large for gestational age neonates in relation to adherence to gestational weight gain recommendations and prepregnancy weight status. Light gray bars represent small for gestational age (SGA), medium gray bars represent appropriate for gestational age (AGA), and darker gray bars represent large for gestational age (LGA) neonates. Prepregnancy weight is categorized as underweight (BMI $\left.<18.5 \mathrm{~kg} / \mathrm{m}^{2}\right)$, normal weight $\left(18.5 \mathrm{~kg} / \mathrm{m}^{2} \leq\right.$ $\left.\mathrm{BMI}<25 \mathrm{~kg} / \mathrm{m}^{2}\right)$, overweight $\left(25 \mathrm{~kg} / \mathrm{m}^{2} \leq \mathrm{BMI}<30 \mathrm{~kg} / \mathrm{m}^{2}\right)$, and obese $\left(30 \mathrm{~kg} / \mathrm{m}^{2} \leq \mathrm{BMI}\right)$. Gestational weight gain adherence based on BMI-specific recommendations per Institute of Medicine 2009 guidelines. Dashed lines indicate expected population burden of SGA and LGA neonates at the 10th and 90th percentiles, respectively.

"adherence" in this context most accurately describes these study conditions. In a previous study of this patient population, we found that counseling about GWG was limited even before guideline changes. ${ }^{23}$ Although women in this study were likely not counseled about GWG using the 2009 guidelines, this study provides an estimate of the contemporary burden of SGA and LGA neonates associated with GWG outside the ranges newly recommended by the IOM.

With an expected normal distribution of birth weights, there will always be neonates who fall at the extremes, some of whom will be constitutionally healthy, whereas others will be pathologically affected. As clearly shown in Figure 2, some groups of women are at significantly higher risk than expected for population burden of having an SGA or LGA neonate. Specifically, underweight women who gain inadequately $(29.1 \%)$ and obese women who gain excessively $(15.2 \%)$ are at significantly higher risk of delivering SGA and LGA neonates, respectively, than the expected $10 \%$.

The strengths of our study include a cohort study design with a large sample of women derived from a clinical care database. This database availed us to examine comorbidities, thus allowing adjustment for underlying medical conditions, such as diabetes and hypertensive disease. Our study sample was drawn from the central Massachusetts region and

Table 2. Small and Large for Gestational Age Neonates in Relation to Prepregnancy Weight Status and Adherence to Gestational Weight Gain Recommendations

\begin{tabular}{|c|c|c|c|c|c|c|}
\hline \multirow{2}{*}{$\begin{array}{l}\text { Prepregnancy weight } \\
\text { status }\end{array}$} & \multirow[b]{2}{*}{$\mathrm{n}$} & \multirow{2}{*}{$\begin{array}{l}\text { Adherence to GWG } \\
\text { recommendations }\end{array}$} & \multicolumn{2}{|c|}{ Small for gestational age neonates } & \multicolumn{2}{|c|}{ Large for gestational age neonates } \\
\hline & & & Crude & Adjusted & Crude & Adjusted \\
\hline \multirow[t]{2}{*}{ Underweight $^{\mathrm{a}}$} & 427 & Inadequate & $2.25(1.22-4.15)$ & $2.51(1.31-4.78)$ & $0.90(0.16-5.04)$ & $0.92(0.16-5.50)$ \\
\hline & & Excessive & $0.69(0.36-1.32)$ & $0.60(0.30-1.20)$ & $2.29(0.70-7.47)$ & $2.99(0.86-10.47)$ \\
\hline \multirow[t]{2}{*}{ Normal weight* } & 5707 & Inadequate & $1.77(1.42-2.20)$ & $1.78(1.42-2.24)$ & $0.58(0.39-0.87)$ & $0.56(0.47-0.73)$ \\
\hline & & Excessive & $0.67(0.54-0.82)$ & $0.59(0.47-0.73)$ & $1.73(1.36-2.20)$ & $1.76(1.38-2.24)$ \\
\hline \multirow[t]{2}{*}{ Overweight* } & 2756 & Inadequate & $0.96(0.6-1.53)$ & $0.99(0.61-1.59)$ & $0.61(0.26-1.43)$ & $0.60(0.25-1.42)$ \\
\hline & & Excessive & 0.71 (0.52-0.97) & $0.64(0.47-0.89)$ & 2.79 (1.81-4.30) & 2.99 (1.92-4.65) \\
\hline \multirow[t]{2}{*}{ Obese* } & 2313 & Inadequate & $0.94(0.60-1.49)$ & $1.01(0.64-1.61)$ & $0.58(0.36-0.93)$ & $0.56(0.34-0.90)$ \\
\hline & & Excessive & $0.82(0.56-1.20)$ & $0.77(0.52-1.14)$ & $1.41(1.01-1.98)$ & $1.55(1.10-2.19)$ \\
\hline
\end{tabular}

Odds ratios (95\% confidence intervals.

adjusted analyses included the following covariates: marital status, parity, smoking, and diabetes.

*Adjusted analyses included following covariates: marital status, race, parity, smoking, diabetes, and hypertension. 
included a patient population that is consistent with the 2008 Census Bureau's report of U.S. racial and ethnic composition. ${ }^{24}$ Our study sample delivery dates ranged from 2006 to 2010 and were representative of the nation's epidemic of obesity, with one fifth of gravidas being obese. ${ }^{25,26}$ These characteristics of our sample potentially enhance the generalizability of our results to populations beyond those of central Massachusetts.

The use of a large clinical database facilitates examination of birth outcomes but has inherent limitations. Evaluation of covariates is limited by the absence of socioeconomic factors, such as education and poverty level. Further, the manner in which data were collected can cause unclear discernment between preexisting vs. gestational conditions (such as diabetes and hypertensive diseases) and, thus, has the potential to differentially affect results. For example, long-standing type 1 diabetes may be associated with vascular disease and SGA neonates, ${ }^{27}$ whereas newly diagnosed GDM is more likely associated with LGA neonates. ${ }^{28}$ A similar limitation was noted by Donahue et al. ${ }^{16}$ when evaluating trends in birth weights in the United States. The association between these comorbidities and SGA or LGA is likely complex and potentially on the causal pathway; for example, weight gain is likely affected by clinical diabetes interventions. However, we performed sensitivity analyses excluding women with these comorbidities, and results were similar (data not shown). An additional limitation is that weight was not measured directly and was instead obtained from data entered into the L\&D EMR by clinical nursing staff when women were in labor. The source of these values was either weight recalled at first prenatal visit or at delivery admission or weight measured at the first prenatal visit. Although a recommended order of prioritized data entry exists, we cannot confirm adherence to the recommended order. Recalled vs. measured weight has been shown by Brown et al. ${ }^{29}$ to produce different results regarding effect of weight gain on newborn size.

Additionally, studies have found that underweight women tend to overestimate ${ }^{30}$ and overweight women tend to underestimate $^{31}$ their prepregnancy weight, and women in this age group generally underestimate their weight and overestimate their heights, ${ }^{32}$ potentially causing inaccurate classification of prepregnancy weight status and, thus, misclassified recommended GWG range. However, self-reported and clinically measured prepregnancy weights have been found to be highly correlated $(r=0.99)^{33}$ and result in the same classification of prepregnancy weight status for $85 \%$ of women. ${ }^{30}$ The proportion of women in our study population who were underweight before pregnancy was small in size compared to other populations, ${ }^{26}$ and, thus, evaluation of this group was limited. Finally, we evaluated adherence by GWG at time of delivery as opposed to gain over specific trimesters or other parameters. Both total gain and rate of gain do not account for the fact that gain at specific pregnancy time points may be more influential on maternal and fetal outcomes, a concept that bears further consideration based on the current available literature. ${ }^{29,34-42}$

\section{Conclusions}

BMI-specific GWG recommendations have been revised recently by the IOM, with the goal of optimizing maternal and neonatal immediate and long-term health outcomes. We found inadequate GWG to be significantly associated with increased odds of SGA for underweight and normal weight women. We also found excessive GWG to be significantly associated with increased odds of LGA for normal weight, overweight, and obese women. Thus, our findings support the updated guidelines with regard to the goal of optimizing birth of appropriate for gestational age neonatal weights. As weight gain during pregnancy is a modifiable factor associated with increased risk of SGA and LGA neonates, further counseling by providers is needed to help women attain guideline adherent weight gain during pregnancy. Further research and public health efforts should be focused on how to assist women in meeting these recommendations.

\section{Acknowledgments}

Material included in this article was reported via oral presentation at the Massachusetts American Congress of Obstetricians and Gynecologists (MA-ACOG) on July 14, 2010, in Waltham, MA, at the American Academy of Pediatrics New England Conference on Perinatal Research on October 19, 2010, in Chatham, MA, and at the Society for Gynecologic Investigation (SGI) annual meeting in March 2011 in Miami Beach, FL, by former chief resident and co-author Anne Garrison, M.D.

\section{Disclosure Statement}

The authors have no conflicts of interest to report.

\section{References}

1. Dietz PM, Callaghan WM, Smith R, Sharma AJ. Low pregnancy weight gain and small for gestational age: A comparison of the association using 3 different measures of small for gestational age. Am J Obstet Gynecol 2009; 201:53.e1-7.

2. Stotland NE, Chen YW, Hopkins LM, Caughey AB. Gestational weight gain and adverse neonatal outcome among term infants. Obstet Gynecol 2006;108:635-643.

3. Arendas K, Qiu Q, Gruslin A. Obesity in pregnancy: Preconceptional to postpartum consequences. J Obstet Gynaecol Can 2008;30:477-488.

4. Hedderson MM, Weiss NS, Sacks DA, et al. Pregnancy weight gain and risk of neonatal complications: Macrosomia, hypoglycemia and hyperbilirubinemia. Obstet Gynecol 2006;108:1153-1161.

5. Wax JR. Risks and management of obesity in pregnancy: Current controversies. Curr Opin Obstet Gynecol 2009;21: 117-123.

6. Sukalich S, Mingione MJ, Glantz JC. Obstetric outcomes in overweight and obese adolescents. Am J Obstet Gynecol 2006;195:851-855.

7. DeVader SR, Neeley HL, Myles TD, Leet TL. Evaluation of gestational weight gain guidelines for women with normal prepregnancy body mass index. Obstet Gynecol 2007;110: 745-751.

8. Finer LB, Henshaw SK. Disparities in rates of unintended pregnancy in the United States, 1994 and 2001. Perspect Sex Reprod Health 2006;38:90-96.

9. Rasmussen $\mathrm{KM}$, Yaktine $\mathrm{AL}$, eds. Weight gain during pregnancy: Reexamining the guidelines. Washington, DC: National Academies Press, 2009.

10. Committee on Nutritional Status During Pregnancy and lactation, Institute of Medicine. Nutrition during pregnancy: Part I: Weight gain, Part II: Nutrient supplments. Washington, DC: National Academies Press, 1990. 
11. Margerison Zilko CE, Rehkopf D, Abrams B. Association of maternal gestational weight gain with short- and long-term maternal and child health outcomes. Am J Obstet Gynecol 2010; 202:574.e1-8.

12. American College of Obstetricians and Gynecologists. Intrauterine growth restriction-ACOG practice bulletin No. 12. Washington, DC: ACOG, 2000

13. American College of Obstetricians and Gynecologists. Fetal macrosomia-ACOG practice bulletin No. 22. Washington, DC: ACOG, 2000.

14. Oken E, Kleinman KP, Rich-Edwards J, Gillman MW. A nearly continuous measure of birthweight for gestational age using a United States national reference. BMC Pediatr 2003;3:6.

15. Kutner MH, Nachtsheim CJ, Neter J, Li W. Applied linear statistical models. New York: McGraw-Hill Irwin, 2005: 608-618.

16. Donahue SM, Kleinman KP, Gillman MW, Oken E. Trends in birth weight and gestational length among singleton term births in the United States: 1995-2005. Obstet Gynecol 2010;115:357-364.

17. Dietz PM, Callaghan WM, Sharma AJ. High pregnancy weight gain and risk of excessive fetal growth. Am J Obstet Gynecol 2009;201:51.e1-6.

18. Control, CFD. Defining childhood overweight and obesity. Available at www.cdc.gov/obesity/childhood/defining.html Accessed December 1, 2010.

19. Alexander GR, Himes JM, Kurman RB, More J, Kogan M. A United States national reference for fetal growth. Obstet Gynecol 1996;87:163-168.

20. Kramer MS, Morin I, Yang $H$, et al. Why are babies getting bigger? Temporal trends in fetal growth and its determinants. J Pediatr 2002;141:538-542.

21. Surkan PJ, Hsieh CC, Johansson AL, Dickman PWN, Cnattingius S. Reasons for increasing trends in large for gestational age births. Obstet Gynecol 2004;104:720-726.

22. Siega-Riz AM, Viswanathan M, Moose MK, et al. A systematic review of outcomes of maternal weight gain according to the Institute of Medicine recommendations: Birthweight, fetal growth, and postpartum weight retention. Am J Obstet Gynecol 2009;201:e39.e1-14.

23. Moore Simas TA, Doyle Curiale DK, Hardy J, Jackson S, Zhang Y, Liao X. Efforts needed to provide Institute of Medicine-recommended guidelines for gestational weight gain. Obstet Gynecol 2010;115:777-783.

24. Available at quickfacts.census.gov/qgd/states/ooooo.html. Accessed July 2, 2010.

25. Chu SY, Callaghan WM, Bish CL, D'Angelo D. Gestational weight gain by body mass index among U.S. women delivering live births, 2004-2005: Fueling future obesity. Am J Obstet Gynecol 2009;200:271.e1-7.

26. Kim SY, Dietz PM, England L, Morrow B, Callahan WM. Trends in prepregnancy obesity in nine states, 1993-2003. Obesity 2007;1:986-993.

27. Haeri S, Khoury J, Kovilam O, Miodovnik M. The association of intrauterine growth abnormalities in women with type 1 diabetes mellitus complicated by vasculopathy. Am J Obstet Gynecol 2008;199:278.e1-5.

28. Esakoff TF, Cheng YU, Sparks TN, Caughey AB. The association between birthweight $4000 \mathrm{~g}$ or greater and perinatal outcomes in patients with and without gestational diabetes. Am J Obstet Gynecol 2009;200:672.e1-4.
29. Brown JE, Murtaugh MA, Jacobs DR Jr, Margellow HC. Variation in newborn size according to pregnancy weight change by trimester. Am J Clin Nutr 2002;76:205-209.

30. Lederman SA, Paxton A. Maternal reporting of prepregnancy weight and birth outcomes: Consistency and completeness compared with the clinical record. Matern Child Health J 1998;2:123-126.

31. Stevens-Simon C, Roghmann KJ, McAnarney ER. Relationship of self-reported prepregnant weight and weight gain during pregnancy to maternal body habitus and age. J Am Diet Assoc 1992;92:85-87.

32. Villanueva EV. The validity of self-reported weight in US adults: A population based cross-sectional study. BMC Public Health 2001;1:11.

33. Oken E, Taveras EM, Kleinman KP, Rich-Edwards JW, Gillman MW. Gestational weight gain and child adiposity at age 3 years. Am J Obstet Gynecol 2007;196:322.e1-e8.

34. Abrams B, Carmichael S, Selvin S. Factors associated with the pattern of maternal weight gain during pregnancy. Obstet Gynecol 1995;86:170-176.

35. Abrams B, Selvin S. Maternal weight gain pattern and birth weight. Obstet Gynecol 1995;86:163-169.

36. Hickey CA, Cliver SP, McNeal SF, Hoffman HJ, Goldenberg RL. Prenatal weight gain patterns and spontaneous preterm birth among nonobese black and white women. Obstet Gynecol 1995;85:909-914.

37. Hickey CA, Cliver SP, McNeal SF, Hoffman HJ, Goldenberg RL. Prenatal weight gain patterns and birthweight among nonobese black and white women. Obstet Gynecol 1996;88: 490-496.

38. Neufeld LM, Haas JD, Grajeda R, Martorell R. Changes in maternal weight from the first to second trimester of pregnancy are associated with fetal growth and infant length at birth. Am J Clin Nutr 2004;79:646-652.

39. Sekiya N, Anai T, Matsubara M, Miyazaki F. Maternal weight gain rate in the second trimester is associated with birth weight and length of gestation. Gynecol Obstet Invest 2007;63:45-48.

40. Siega-Riz AM, Adair LS, Hobel CJ. Maternal underweight status and inadequate rate of weight gain during the third trimester of pregnancy increases the risk of preterm delivery. J Nutr 1996;126:146-153.

41. Spinillo A, Capuzzo E, PIazzi g, Ferrari A, Morales V, DiMario M. Risk for spontaneous preterm delivery by combined body mass index and gestational weight gain patterns. Acta Obstet Gynecol Scand 1998;77:32-36.

42. Strauss RS, Dietz WH. Low maternal weight gain in the second or third trimester increases the risk of intrauterine growth retardation. J Nutr 1999;129:988-993.

Address correspondence to: Tiffany A. Moore Simas, M.D., M.P.H., M.Ed. Divisions of Research and General Obstetrics and Gynecology Department of Obstetrics and Gynecology University of Massachusetts Medical School/ UMass Memorial Health Care Memorial Campus 119 Belmont Street, Jaquith Build. Room 4053 Worcester, MA 01605

E-mail: tiffanya.mooresimas@umassmemorial.org 